\title{
EDUCATION INNOVATION THROUGH MATERIAL INNOVATION IN PRIMARY EDUCATION: THE ‘GROW- IT-YOURSELF' WORKSHOP
}

\author{
Lore BROSENS and Marina EMMANOUIL \\ Ghent University, Department of Industrial Systems Engineering and Product Design
}

\begin{abstract}
In recent years more STEM (Science, Technology, Engineering and Mathematics) topics have been incorporated in mainstream public education. Although the benefits of STEM instruction are broadly recognised in secondary school curricula, STEM topics in primary education are rather limited, leaving a gap in manipulative skills building and in preparation processes for the next school level. This paper reflects on the outcomes of a design workshop attended by 12 primary school students ( 9 to 12 years old) in Belgium. Mycelium, a fungi-based natural material now used in innovative sustainable applications, served as a means to introduce early learners engineering basics through selfmade learning tools. Students grew their own 3-D structures to build a 'Grow-It-Yourself' biodegradable playground using mycelium as a primary source. The paper stems from an in-progress research that investigates the opportunities of how mycelium as a material innovation can be used as a medium to create innovation in primary education through a learning-by-design approach. Reflections on the workshop's instructional guidelines are included along with an extension of the call for support for primary school teachers delivering STEM topics in their classes.
\end{abstract}

Keywords: Learning by doing/design, STE(A)M-education, primary education, creativity, workshop guidelines, sustainability, mycelium

\section{INTRODUCTION}

Contemporary pedagogical literature posits that in order to be able to ask critical questions and create ingenious solutions to everyday problems, STEM education needs to be fully integrated in mainstream school curricula [1]. This type of education aims to teach students a number of science, technology, math and engineering topics in order to increase literacy in general science subjects that would ultimately create more skilled individuals for science-driven jobs of the future [2][3][4]. Most importantly STEM education is argued to contribute to the development of problem solving skills and fostering critical thinking [5]. In the early 2000's a concentrated effort to create awareness for a further implementation of STEM topics into public education was initiated by a number of educators and visionaries [5]. Garner et al. [6] claimed that STEM-driven education lead to a range of socialcognitive competencies that are considered $21^{\text {st }}$ century life skills. In order for STEM education to fulfil its promises it is essential not only to acquaint students to different science disciplines but to do so through enabling and invoking creativity [2]. Mainstream education can find a partner in achieving this through the various methods practised in design education that specifically support the development of creative and critical thinking skills. Indeed, a recent development that shows such coalition is becoming a reality, is the incorporation of a new factor in the classic STEM model. The new acronym STEAM (with the 'A' standing for Art) involves topics originating from liberal, studentcentred pedagogical models that explore creativity and learning-by-doing practices. It is argued that when students learn through creativity-inductive, hands-on experiences satisfaction, motivation and memorability is increased especially for students with learning difficulties [7]. The shift from STEM to STEAM was somewhat expected since STEM topics typically lend themselves to learning-by-doing experiences [8]. For instance, to solve a technical problem a physical interaction with the technology at stake is often unavoidable. The benefits of STEM education especially with a creative dimension are highlighted in commercial campaigns by private enterprises. Initiating excitement and love for 
science is the marketing selling point of several companies that produce STEM educational toolkits and toys for children and young adolescents (5-16 years old). In terms of costs, these products come in the range of $£ 15.40$ for a monthly subscription to a KiwiCo toolbox and $£ 279.99$ for a computer kit from Kano [9][10]. Even though the availability of these outside of formal educational institutions could potentially enrich and support STEM goals, they pose certain pedagogical and ethical concerns regarding democratisation of education in an open source society.

Returning back to school education, a growing number of arguments are in support of an early introduction of a learning-by-doing approach and of STEAM education in primary school instruction. These claims relate to manipulative skills development and smoother preparation for transitioning in secondary education. With respect to the first issue, it is argued that the need for hands-on activities is bigger in primary schools due to children's limited manipulative skills at that age [3]. Incompetence in motor skills in primary school may be carried through in secondary school [3]. Regarding the second issue, exposure to STEM topics at an earlier stage is shown to help prepare young children build basic science competences for later years. Sharapan [11] supports that introduction of STEAM in primary education can create a proper basic understanding of math and science related knowledge to cognitively support the more advanced scientific principles in secondary education. Also, developing critical thinking skills in primary education level offers opportunities for young children to prepare the ground for growing a critical mindset on pressing world challenges, such as acting in a more responsible way towards the environment as an adult.

Early introduction of science basics in primary education comes with many advantages as shown in literature. However, despite the promising steps in recent years, integration of STEM/STEAM in public schools is still lacking behind its full potential [1]. Introduction of new pedagogies in schools, such as student-centred learning is hindered due to a number of reasons. Implementation of new ways of teaching is challenged by teachers' subjective beliefs about what K-12 pedagogy should entail, and on the level of support teachers get when introducing STEM topics to their students [12]. On the latter, especially primary school teachers have reported that they do not feel supported enough to introduce science related topics [8]. Also, schools often lack the necessary resources to be used for introducing science subjects in a creative and playful way appropriate for the respective level of education [8]. Therefore, a question that still awaits an answer is: How can primary teachers be practically assisted in introducing STEM topics in their classes? This research seeks to address this question by examining how material innovation could become the driving force for education innovation in primary schools. The following section presents the general framework of a 'learning by doing' instructional model and basic theoretical underpinnings on 'learning by design'. The paper ends with a description of the workshop designed to assist teachers implement scientific topics in a creative, fun way and with a sustainability dimension.

\section{LEARNING BY DOING / LEARNING BY DESIGN}

Confucius's infamous line, "I hear and I forget. I see and I remember. I do and I understand" influenced many educational curricula across space and time. Jean Piaget's epistemological learning theory of Constructivism regards knowledge as being constructed by the student [13][14]. Such viewpoint concerns the ways mental constructions get formed by the individual learner. In extending this cognitive framework into the physical world, Papert's model considers learning to be a reconstruction rather than a transmission of knowledge that is 'most effective when part of an activity is experienced by the learner [while] constructing a meaningful product' [15]. With the phrase "education with inert ideas is not only useless: it is, above all things, harmful" Lesgold [16] underlined the importance of a 'learning by doing' model of instruction at the end of the $20^{\text {th }}$ century. The process of learning involves creating patterns and connections in our brains that are stimulated by what we pick up with our senses, making learning through interactive means more effective [16]. In experiential learning students draw their own thoughts and ideas through creative hands-on experimentation and make social objects that others can see and critique. The workshop sought to create space for students to apply hands-on experimentation in order to construct a tangible object, a playground.

Learning by doing, or experiential learning takes the shape of problem-based learning and 'learning by design' models of instruction [17]. Currently popular in diverse fields such as IT, business and medicine [18][19], design thinking is often described as a methodology in tackling 'wicked problems', that is, complex and complicated problems that cannot be analysed and fully understood by rational, 
scientific ways [20][21]. Instead, this kind of problems need to be reframed and analysed iteratively and require an ability to move from concrete to abstract modes of thinking, as well as, from divergent (associated to imagination and intuition, or 'making' choices) to convergent processes (associated with rational thought, or 'creating' choices). According to Owen [22] design thinking involves the construction of knowledge that is situated between two important cognitive processes: analysis and synthesis, in other words 'breaking problems apart and putting ideas together'. In its basic idea, the workshop contained to a certain extent both analytic processes by recognising and confronting several interdependent challenges (e.g., how mushrooms grow) and the synthetic processes (making), in the sense of creating visual representations of components of a playground. In learning by design, deep learning is achieved by creating an object that requires understanding and application of knowledge [17]. As design is an iterative process, students are engaged in a number of technical and cognitive activities in a mode of revision that require collaboration and reflection. Both higher and lowerachieving students display strong evidence of progress in learning and applying key concepts in their design work [23][24].

\section{THE ‘GROW-IT-YOURSELF’ DESIGN WORKSHOP}

\subsection{Background}

The idea of implementing a design workshop is the outcome of the first author's undergraduate project during an engineering design course about investigating possible applications using mycelium. The idea to implement this material/technology into the material world of children emerged from the basic observation of children getting dissatisfied by their (often plastic) toys every few weeks, feeding the throw away economy and potentially harmful for the environment. The workshop aimed at broadening students' worldview through building an awareness of the role of materials/technologies play in creating a sustainable future. The interaction with the material properties of mycelium would offer a first acquaintance with biology and sustainability. Mycelium is created underground (by the growing structures of mushrooms), relatively strong and adaptable to nearly any form. It is essentially a biomaterial with low energy demands to grow and biodegradable, making it a sustainable material, a 'material of the future' [25]. The idea of a mycelium-grown playground could offer a continuous cycle experience in which students can create a playground that would compose naturally after having served its educational purpose. A self-made video of some basic information on what mycelium is and how it is used to create products is available at: https://youtu.be/lqBgtP9Hnbs. The workshop was organised through communication with the director of the school and a teacher, who provided feedback. Twelve students were placed in three groups of four to get some preliminary responses on the effectiveness of different instruction types. All participants came from three different age and six class groups. Each group had mixed-level students (year grades 4 to 6, ages 9 to 12) and mixed ability with participants been identified by the teachers as 'gifted' and some with learning difficulties.

\subsection{Procedure}

In order to successfully introduce a subject through a learning-by-doing experience it is customary for students to follow a certain number of activities in sequence that would lead to thought processes in a specific order. This is necessary in order for them to effectively learn from the experience and ask relevant questions. Roberts [26] developed the idea of dynamic learning by creating a frame for designing a workshop using the multiple senses approach, the E.E.L.D.R. This design framework involves the following steps: Enrol, Experience, Label, Demonstrate, and Review. This model was applied in the mycelium workshop by first creating a bigger picture and letting children experience what the technology is and what it can do. During the Label phase children would learn more about the biology elements of mycelium and how mushrooms grow. When [D]emonstrating what needs to happen children would read the instructions together with the teacher. Finally in the Review section of the workshop children would independently work on their own project. Following on this structure, students were asked to create geometric shapes in Thinkercad, an online open-source CAD program [26]. Afterwards the shapes were created out of cardboard and sliced into 3-D puzzles. Students then got engaged with 3-D thinking and built the previously made shapes in real life (Fig. 1-4). Afterwards students would fill the cardboard shapes with a mycelium (the mushroom material) and the substrate (the food the mushroom feeds on). The shapes made of mycelium could then grow and dry within 
three weeks. The blocks are lightweight and safe to stack on top of each other. The mycelium is waterproof, not poisonous and starts composing normally after three weeks of usage.
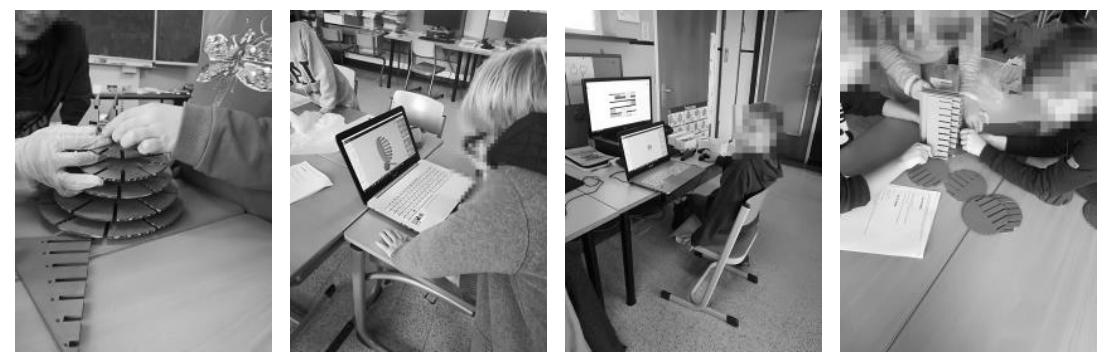

Figure 1-4. Creating and building 3-D shapes

\subsection{Instructions and preliminary reflections}

The workshop stood as an opportunity to put in practice three basic guidelines assigned to instructions: clear communication, application of constraints, and target outcome awareness. First, research has shown that the less information instruction users get the more uniform the outcome of the instructions would be [27][28]. Instructions should be simple and contain only the necessary information. Excessive amounts of instructions are a problem especially for students with special learning needs [28]. A suggested method for clear communication when giving instructions and for maintaining the creative input of the users during the process is the "cookbook approach" [27]. This has a standardised form of formal and clear instruction communication often in a list format. A cooking recipe usually contains written information and some visuals (e.g., the dish outcome). Yet, how easily can young children deal with both text and images when following instructions? This basic question is taken up in the following paragraphs. Second, literature on creativity posits that the feeling of creative accomplishment is influenced by the amount of constraints given in the instructions [29]. One would think that adding constrains to an educational activity may make participants feel restricted, yet, it has been shown that by providing constraints, skill requirements are lowered [29]. Constraints were included in the workshop to guide children through the learning process. Third, whether (or not) a target outcome is given has an enormous impact on how participants perceive a workshop [29]. When introducing a new topic, students should be provided with a greater view of the subject in order to frame the experience better and be able to make connections in their brains easier [26]. The end result of the workshop was shown from the start. Lastly, research in children's apprehension of instructions has shown that children from the age of 8 and up are able to think in problem solving terms and are capable of following a guideline when trained in these skills [30]. Also, when instructions are compatible with their natural exploratory mode younger children can follow the provided guidelines as good as more mature students [30]. However, it is also stressed that the age and stage of a child's development are not fully determining whether children are able to follow instructions or not [30].

A basic pilot study was made to examine how these recommendations would work in the case of giving instructions during the workshop. The latter, acted in a way as an incubator to investigate first, issues related to 'clear communication' (for 9-12 year olds), and second, how instructions are perceived to affect their sense of creativity. For the first issue, a basic question was formulated: is the medium of instruction (text or visuals) playing a role in understanding instructions? For this inquiry, one type of instructions (group 1) included a list (text only) composed of imperative sentences with one central action per activity (fig. 5). Participants were asked to read this list by themselves and could be assisted by the teacher, if needed. The second type of instructions (group 2) was visual-based, i.e., pictures with cues (fig. 5). Visual cues took the form of colour-matching dots indicating which slot connects with another slot to form a structure/object. This method is often used in children's DIY toolkits, e.g., Christmas tree constructions. A picture of the end result was included in both types of instructions. For the second question, students were asked whether they felt creative when following on instructions and about their sense of accomplishment for the end result. The experiment of the two instruction types led to some interesting results. Group 1 performed well in reaching the desired outcome and followed the steps (as group 2 did) but completed the task faster than group 2. Students mentioned that they felt very accomplished afterwards but restricted when following such detailed instructions. Most students in group 1 said that they did not see the need to think creatively. Group 2 performed as good as group 1 in reaching the end result, but they needed more time to complete all 
steps. Not all group 2 members were as quick to understand the instructions as their team peers. Unfamiliarity with the dot method and the 3-D software (or due to high allocation of cognitive resources for these activities) may have made apprehension of the activity harder for some students. Group 2 had a good experience during the workshop but not all of them felt accomplished. However, when asked whether they were happy with their own ability to add creative output, they (group 2) felt very accomplished with their own contribution.
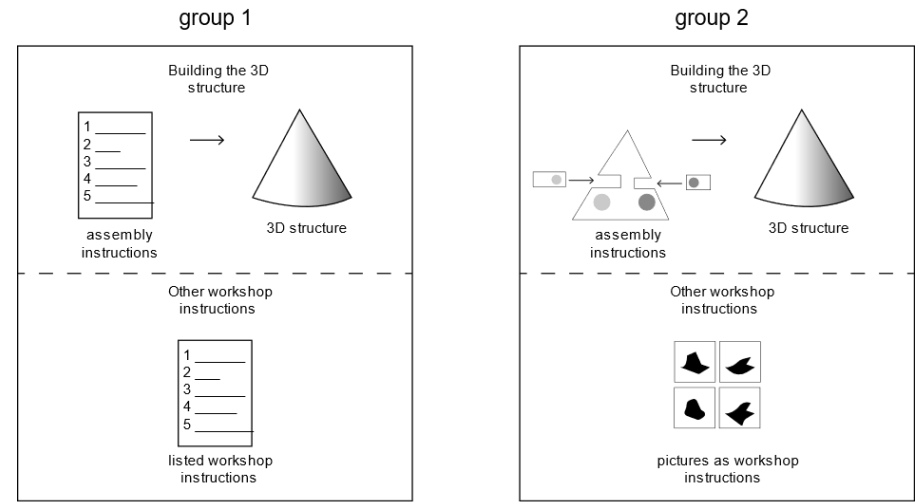

Figure 5. Instruction media for the two groups

Some preliminary reflections at this point could be made. Giving all instructions at once may lead to confusion and distractions. Division of sections and information in consecutive moments during the workshop might have offered better outcomes. Additionally, leaving some actions out of the list could potentially serve as stimulant for students to take initiatives and induce creative thinking. All participants created relatively easy 3-D shapes when using CAD. This might be due to their unfamiliarity with this digital tool and its advanced options. If further experimentation were desired (e.g., for producing more elaborate shapes), prior training would be useful. Lastly, due to the time requirements for mycelium to grow (3 weeks), immediate feedback on students' constructions was not possible. Since immediate feedback is a critical aspect of a learning-by-doing approach [31], making a comeback session after three weeks, or/and have examples of dried mycelium pieces on the day of the workshop as benchmarks, could be feasible options.

\section{THOUGHTS AND FUTURE WORK}

The purpose of the design workshop was to introduce mycelium to early learners and through this innovative material/technology to get acquainted with biology, engineering basics and sustainable actions. In practice, students designed and grew their own 3-D structures with mycelium following two types of instructions. The pilot study in its basic format showed that text-only instructions might create fast outcomes but restrict space for creative initiatives. On the other hand, visual-only instructions appear to have allowed students' creative initiatives but require extra time for apprehension of these guidelines. Exploring further the connection of instructions and creativity during a learning by doing/design workshop in quasi-experimental conditions is the aim of this ongoing research towards a better understanding of educational practices for all-ability students in primary school environments.

\section{REFERENCES}

[1] Zollman A. Learning for STEM Literacy: STEM Literacy for Learning. School Science and Mathematics, 2012, 112(1), 12-19.

[2] Cook K.L. and Bush S.B. Design thinking in integrated STEAM learning: Surveying the landscape and exploring exemplars in elementary grades. School Science and Mathematics, 2018, 118, pp. 93-103.

[3] Fadzil H.M. and Saat R.M. Enhancing STEM education during school transition: Bridging the gap in science manipulative skills Eurasia Journal of Mathematics, Science and Technology Education, 2014, 10(3), pp. 209-218.

[4] Karahan, E. and Canbazoglu-Bilici S. and Unal A. Integration of Media Design Processes in Science, Technology, Engineering, and Mathematics (STEM) Education Eurasian Journal of Educational Research, 2015, 60, pp. 221-240. 
[5] Purzer Ş. and Shelley M. Engineering Education in Elementary and Secondary Schools. International journal of education in mathematics, science and technology, 2018, 6(4), pp. I-V.

[6] Garner P.W., Gabitova N., Gupta A. and Wood T. Innovations in science education: infusing social emotional principles into early STEM learning. Cultural Studies of Science Education, 2017, 13(4), pp. 1-15

[7] Thuneberg H.M., Salmi H.S. and Bogner F.X. How creativity, autonomy and visual reasoning contribute to cognitive learning in a STEAM hands-on inquiry based math module Thinking stills and creativity, 2018, 29, pp. 153-160.

[8] Jamil F.M., Linder S.M. and Stegelin D.A. Early Childhood Teacher Beliefs About STEAM Education After a Professional Development Conference Early Childhood Education Journal., 2018, 46(4), pp. 409-417.

[9] KiwiCo inc. KiwiCo. Available: https://www.kiwico.com/ [Accessed on 2019, 10 February] (2017) 06 June.

[10] Kano, Kano. Available: https://kano.me/uk. [Accessed on 2019, 10 February] (2016) 06 January.

[11] Sharapan H. From STEM to STEAM: How Early Childhood Educators Can Apply Fred Rogers' Approach Young Children, 2012, 67(1), pp. p36-40.

[12] Hains B. J. and Smith B. Student-Centered Course Design: Empowering Students to Become Self-Directed Learners. Journal of Experiential Education, 2012, 35(2), 357-374.

[13] Papert S. Constructionism, 1991 (Ablex Publishing Corporation).

[14] Cakir M. Constructivist approaches to learning in science and their implications for science pedagogy: A literature review. International Journal of Environmental \& Science Education, 2008, 3(4), 193-206.

[15] Sabelli N. Constructionism: A New Opportunity for Elementary Science Education. Division of Research on Learning in Formal and Informal Settings, 2008, 193-206.

[16] Lesgold A.M. The Nature and Methods of Learning by Doing. American Psychologist, 2001, 56(11), 964-973.

[17] Barron B. and Darling-Hammond L. Teaching for Meaningful Learning: A Review of Research on Inquiry-Based and Cooperative Learning. Powerful Learning: What We Know About Teaching for Understanding, 2008, 1-15.

[18] Dorst K. The core of 'design thinking' and its application. Design Studies, 2011, 32(6), 521-532.

[19] Renard H. Cultivating Design Thinking in Students through Material Inquiry. International Journal of Teaching and Learning in Higher Education, 2014, 36(3), 414-424.

[20] Buchanan R. Wicked Problems in Design Thinking. Design Issues, 1992, 8(2), 5-21.

[21] Cross N. Designerly Ways of Knowing: Design Discipline versus Design Science. Design Issues, 2016, 17(3), 49-55.

[22] Owen C. Design Thinking: Notes on its Nature and Use. Design Research Quarterly, 2007, 2(1), $16-27$

[23] Fortus D., Dershimer R.C., Krajcik J., Marx R.W. and Mamlok-Naaman R. Design-based science and student learning. Journal of Research in Science Teaching, 2004, 41(10), 1081-1110.

[24] Wang F. and Hannafin M.J. Using design-based research and research of technology-enhanced learning environments. Aera, 2004, 1-6.

[25] Bayer E. Eben Bayer: Are Mushrooms the New Plastic? TED, 2010

[26] Roberts J.W. Beyond Learning by Doing: The Brain Compatible Approach Journal of Experiential Education, 2002, 25(2), pp. 281-285.

[27] Dalton M.A., Desjardins A. and Wakkary R. From DIY tutorials to DIY recipes. In ACM conference on Human factors in computing systems, CHI EA '14, Toronto, Canada, April 2014, pp. $1405-1410$.

[28] Sy J.R., Donaldson J.M., Vollmer T.R. and Pizarro E. An evaluation of factors that influence children's instruction following. Journal of Applied Behaviour Analysis, 2014, 47(1), 101-112.

[29] Dahl D.W. and Moreau C.P. Thinking Inside the Box: Why Consumers Enjoy Constrained Creative Experiences. Journal of Marketing Research, 2017, 44(3), pp. 357-369.

[30] Vlietstra A.G. Children's Responses to Task Instructions: Age Changes and Training Effects. the Society for Research in Child Development, 2018, 53(2), 534-542.

[31] Gelderblom H. and Kotzé P. Ten design lessons from the literature on child development and children's use of technology. In Interaction Design and Children, IDC 2009, Como, Italy, June 2009, pp. 52-60. 